Patrick Merz Paranhos

Localização em Ambientes Externos através da Fusão de Sensores GPS e Inercial por um Filtro de Kalman

Dissertação apresentada como requisito parcial para obtenção do título de Mestre pelo Programa de PósGraduação em Engenharia Mecânica da PUC-Rio.

Orientadores: Marco Antonio Meggiolaro Álvaro de Lima Veiga Filho 
Patrick Merz Paranhos

\section{Localização em Ambientes Externos através da Fusão de Sensores GPS e Inercial por um Filtro de Kalman}

Dissertação apresentada como requisito parcial para obtenção do título de Mestre pelo Programa de PósGraduação em Engenharia Mecânica da PUC-Rio. Aprovada pela Comissão Examinadora abaixo assinada.

Prof. Marco Antonio Meggiolaro Orientador Departamento de Engenharia Mecânica - PUC-Rio

Prof. Álvaro de Lima Veiga Filho Co-Orientador Departamento de Engenharia Elétrica - PUC-Rio

Prof. Mauro Speranza Neto Departamento de Engenharia Mecânica - PUC-Rio

Prof. Luciano Luporini Menegaldo Instituto Militar de Engenharia

Prof. José Eugenio Leal Coordenador Setorial do Centro Técnico Científico - PUC-Rio 
Todos os direitos reservados. É proibida a reprodução total ou parcial do trabalho sem autorização da universidade, do autor e do orientador.

\section{Patrick Merz Paranhos}

Engenheiro de Controle e Automação formado pela PUCRio em 2007.

Ficha Catalográfica

Paranhos, Patrick Merz

Localização em ambientes externos através da fusão de sensores GPS e Inercial por um filtro de Kalman / Patrick Merz Paranhos ; orientadores: Marco Antonio Meggiolaro, Álvaro de Lima Veiga Filho. - 2009.

179 f. : il. (color.) ; $30 \mathrm{~cm}$

Dissertação (Mestrado em Engenharia Mecânica)-Pontifícia Universidade Católica do Rio de Janeiro, Rio de Janeiro, 2009.

Inclui bibliografia

1. Engenharia mecânica - Teses. 2. Filtro de Kalman. 3. GPS. 4. Sensor Inercial. 5. Robôs móveis em ambiente externo. 6. Fusão de sensores. 7. Localização. I. Meggiolaro, Marco Antonio. II. Veiga Filho, Álvaro de Lima. III. Pontifícia Universidade Católica do Rio de Janeiro. Departamento de Engenharia Mecânica. V. Título. 


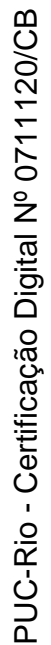

Dedico esta dissertação a minha família que sempre se manteve ao meu lado. 


\section{Agradecimentos}

aos Professores Doutores Marco Antonio Meggiolaro e Álvaro de Lima Veiga Filho pela orientação preciosa;

ao Ney Robinson por me acolher no projeto do Robô Ambiental Híbrido;

aos meus amigos de Laboratório no CENPES por sua amizade e ajuda;

a meu amigo Ricardo Saavedra por sua paciência e ajuda com as minhas incontáveis duvidas de português;

à FAPERJ pela bolsa de estudos. 


\section{Resumo}

Paranhos, Patrick Merz; Meggiolaro, Marco Antonio. Localização em Ambientes Externos através da Fusão de Sensores GPS e Inercial por um Filtro de Kalman. Rio de Janeiro, 2009. 179p. Dissertação de Mestrado - Departamento de Engenharia Mecânica, Pontifícia Universidade Católica do Rio de Janeiro.

Um dos problemas em soluções que envolvam mobilidade é estimar a posição do robô com precisão. Em ambientes externos, o sensor GPS é o mais comumente utilizado, pois o mesmo fornece uma posição global, porém existe uma imprecisão que é superior a alguns metros, além de depender da visibilidade aos satélites. Outra solução é utilizar um sensor inercial, que no início da operação apresenta uma boa precisão, porém o erro de posicionamento cresce ilimitadamente por ser calculado através da integral dupla das acelerações e velocidades angulares medidas. $\mathrm{O}$ presente trabalho desenvolve um sistema de localização de robôs móveis em ambientes externos. As soluções do posicionamento via GPS e via sensor inercial são combinadas através de um filtro de Kalman, reduzindo a incerteza da obtenção da posição. $\mathrm{O}$ equacionamento e duas implementações distintas do filtro de Kalman serão apresentadas. Uma implementação clássica e uma versão estendida para sensores inerciais de baixa qualidade, a qual utiliza a orientação fornecida por bússolas na filtragem. Através de experimentos e simulações será demonstrada a eficácia da localização através do filtro de Kalman e a melhora na performance do mesmo quando utilizado a implementação estendida em comparação a clássica.

\section{Palavras-chave}

Filtro de Kalman; GPS; Sensor Inercial; Robôs Móveis em Ambiente Externo; Fusão de Sensores; Localização 


\section{Abstract}

Paranhos, Patrick Merz; Meggiolaro, Marco Antonio (Advisor). Localization in External Environments through GPS/INS Kalman Filter. Rio de Janeiro, 2009. 179p. MSc. Dissertation - Departamento de Engenharia Mecânica, Pontifícia Universidade Católica do Rio de Janeiro.

One of the problems with solutions that involve mobility is to accurately estimate the robot's position. In an outdoor environment, the GPS sensor is the most commonly used method because it provides a global position, but with an error margin that is greater than just a few meters, and creates a dependency on the visibility of the satellites. Another solution is to use an inertial sensor, which at the beginning of the operation shows good accuracy, but the positioning error grows indefinitely because it is calculated by a double integral of acceleration and angular velocity measures. This work develops a system for localization of mobile robots in outdoor environments. The positions are estimated via GPS and inertial sensors, combined using a Kalman filter, reducing the uncertainty. The equations and two distinct implementations of the filter will be presented. A classical implementation and an extended version for low-grade inertial measurement units, which utilizes the orientation given by compasses in the filtering process. The effectiveness of the Kalman filter navigation is verified through experimental and simulation results. The performance gain of the extended filter in comparison to the classic is also verified.

\section{Keywords}

Kalman Filter; GPS; Inertial Sensor; Mobile Robot; Localization in External Environment; Sensor Fusion; 


\section{Sumário}

1 Introdução 19

1.1. Motivação 22

1.2. Objetivo 23

1.3. Justificativa 23

1.4. Revisão Bibliográfica 24

1.5. Estrutura da Dissertação 25

2 Sensores $\quad 27$

2.1. Erro 27

2.2. Sensores Proprioceptivos 28

2.2.1. Odômetros 29

2.2.2. Sensores Inerciais 29

2.3. Sensores Exteroceptivos 36

2.3.1. Energia Acústica $\quad 37$

2.3.2. Energia Eletromagnética 38

2.3.3. Medição por Tempo de Vôo 39

2.3.4. Medição por Diferença de fase 40

2.3.5. Medição por Variação de Frequência 41

2.3.6. Medição por Triangulação 41

2.3.7. Medição por Velocidade Relativa 43

2.3.8. LIDAR 43

2.3.9. Sonar 45

2.3.10. Radar de Milímetro 45

2.3.11. Bússolas 47

2.3.12. Sensores Baseados em Visão 48

2.3.13. Sistema de Satélites para Navegação Global 48

2.4. GPS 50

2.4.1. Receptores 51 
2.4.2. Erro 52

2.4.3. Disponibilidade Seletiva (SA) 53

2.4.4. lonosfera 53

2.4.5. Troposfera 54

2.4.6. Múltiplos Caminhos $\quad 54$

2.4.7. Dados Ephemeris $\quad 55$

2.4.8. Relógio do Satélite $\quad 55$

2.4.9. Resolução e Ruído do Receptor 55

2.4.10. Diluição de Precisão (DOP) 56

2.4.11. Sistema Diferencial de Posicionamento de Satélites

3 Filtro de Kalman Discreto $\quad 59$

3.1. Sistemas Lineares $\quad 59$

3.2. Estimação Probabilística 60

3.3. Filtro de Kalman 61

3.4. Exemplo 64

3.4.1. Simples Posicionamento em 1 Dimensão 64

3.4.2. Ajuste do Ruído da Medida 66

3.5. Sistemas Não Lineares 68

3.6. Filtro de Kalman Estendido 69

3.6.1. Linearização 69

3.6.2. Equação do Filtro de Kalman Estendido 73

4 Navegação Inercial (INS) $\quad 75$

4.1. Modelo da Terra 75

4.2. Sistemas de Coordenadas 76

4.2.1. Sistema Centrado e Fixo na Terra (ECEF) 77

4.2.2. Sistema Inercial Centrado na Terra (ECI) 78

4.2.3. Sistema Localmente Tangente (ENU) 79

4.2.4. Sistema Wander-Azimuth (NAV) 80

4.2.5. Coordenadas do Corpo 80

4.2.6. Coordenada de Medida 81

4.3. Navegação Inercial $\quad 82$ 
4.3.1. Bloco 0 - Transformação das Coordenadas de Medida para as Coordenadas do Corpo. 84

4.3.2. Bloco 1 - Aceleração $A^{n}$.

4.3.3. Bloco 2 - Velocidade $V^{n}$.

4.3.4. Bloco 3 - Velocidade angular do sistema NAV relativo a $\operatorname{ECEF}\left(\rho^{\mathrm{n}}\right)$.

4.3.5. Bloco 4 - Posição Angular $\left(\mathrm{C}_{\mathrm{e}}{ }^{\mathrm{n}}\right)$

4.3.6. Bloco 5 - Velocidade Angular da Terra expressa nas Coordenadas NAV $\left(\Omega^{\mathrm{n}}\right)$.

4.3.7. Bloco 6 - Velocidade Angular do sistema NAV com relação ao sistema $\mathrm{ECl}\left(\mathrm{w}^{\mathrm{n}}\right.$ in). $\quad 98$

4.3.8. Bloco 7 - Orientação $\left(\mathrm{C}_{\mathrm{b}}{ }^{\mathrm{n}}\right)$. 99

4.3.9. Condições Iniciais 100

4.4. Erro na Navegação Inercial 101

4.4.1. Erro na Posição Angular $\left(\delta \mathrm{C}_{\mathrm{e}}{ }^{\mathrm{n}}\right)$

4.4.2. Erro na Orientação $\left(\delta C_{b}{ }^{n}\right)$

4.5. Propagação do Erro 105

4.5.1. Erro da Derivada da Posição Angular $\left(\delta \mathrm{C}_{\mathrm{e}}{ }^{\mathrm{n}}\right)$.

4.5.2. Erro na Latitude $(\delta \Phi)$, Longitude $(\delta \lambda)$ e Wander $(\delta \alpha)$. 108

4.5.3. Erro na Derivada de Velocidade $(\delta \mathrm{V})$

4.5.4. Erro na Gravidade ( $\delta \mathrm{g}) \quad 109$

4.5.5. Erro na Aceleração $\left(\delta A^{n}\right) \quad 110$

4.5.6. Erro na Velocidade Angular do sistema NAV relativo a $\operatorname{ECEF}\left(\delta \rho^{\mathrm{n}}\right) \quad 111$

4.5.7. Erro na Altura ( $\delta \mathrm{h}) \quad 111$

4.5.8. Erro na Orientação $\left(\delta \mathrm{C}_{b}{ }^{n}\right)$

5 Equacionamento do Filtro de Kalman 114

5.1. Vetor de Estado $(x)$

5.2. Matriz de Observabilidade Clássica $(H)$

5.3. Matriz de Observabilidade Orientação $(H)$

5.4. Matriz de Dinâmica do Sistema (F) 120

5.5. Vetor de Entrada (u) 124 
5.6. Ruídos da Medida 125

5.7. Ruídos do Processo 125

6 Simulações 126

6.1. Descrição da Simulação 126

$\begin{array}{ll}\text { 6.2. Entrada } & 127\end{array}$

6.3. Modelo do Sensor Inercial (IMU) 128

6.4. Modelo do GPS 130

6.5. Navegação Inercial (INS) 131

6.6. Filtro de Kalman do GPS/INS 131

6.7. Resultados da Simulação 135

6.7.1. RMS 135

6.7.2. Posição 136

$\begin{array}{ll}\text { 6.7.3. Velocidade Linear } & 141\end{array}$

6.7.4. Orientação 142

6.7.5. Aceleração Linear 146

$\begin{array}{ll}\text { 6.7.6. Velocidade Angular } & 147\end{array}$

6.8. Resultados da Simulação de Falha do Sinal de GPS 148

7 Experimentos 152

7.1. Experimento na Floresta Amazônica 153

7.2. Ambiente Visualmente Reconhecível 154

7.3. Resultado Experimental 155

7.4. Resultados dos Experimento na Amazônia 156

7.5. Resultado Experimental no Ambiente

Visualmente Reconhecível 160

7.6. Simulação de Falha no sinal do GPS no

Experimento 165

$\begin{array}{ll}\text { 7.7. Resultados de Outras Literaturas } & 169\end{array}$

8 Conclusões 172

$\begin{array}{ll}\text { Bibliografia } & 174\end{array}$ 
Anexo A

Anexo B

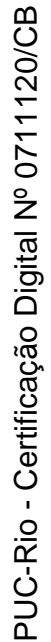




\section{Lista de figuras}

Figura 1 - Robô GP8 da Seegrid. 20

Figura 2 - Míssil de cruzeiro (Taurus KEPD 350). 20

Figura 3 - Rover para operações em solo marciano (MER-A). 21

Figura 4 - Diagrama 22

Figura 5 - Robô Ambiental Híbrido. 23

Figura 6 - Erros comuns entre entrada e saída: (a) bias; (b) fator de escala; (c) não-linearidade; (d) assimetria; (e) zona morta; (f) quantização. 28

Figura 7 - Sensor inercial Guimbale (BOSE, et. al., 1982) 30

Figura 8 - Sensor inercial Strapdown (BOSE, et. al., 1982) 30

Figura 9 - (a) Acelerômetro Mecânico. (b) Piezoelétrico.

(DUDEK; JENKIN, et. al., 2008) 31

Figura 10 - Girômetro "Ring Laser" 32

Figura 11 - Girômetro de fibra óptica 32

Figura 12 - Girômetro Mecânico.

Figura 13 - Girômetro por efeito de Coriolis. 33

Figura 14 - A não detecção de uma fenda devido à divergência

da onda sonora. 38

Figura 15 - Detecção do ambiente mais próximo do que este realmente se encontra. 38

Figura 16 - Espectro da energia eletromagnética 38

Figura 17 - Triangulação 1D 42

Figura 18 - Padrão estruturado de luz para triangulação em

2 dimensões, (RAMAMOORTHI et. at.) 43

Figura 19 - LIDAR mapeando em duas dimensões. 44

Figura 20 - Resultado de uma varredura utilizando um sonar $\quad 45$

Figura 21 - Varredura feita por um radar de milímetro.

(SCHEDING; BROOKER; HENNESSY; BISHOP et. al., 2002) 46

Figura 22 - Constelação de Satélites. (Brock University 
Map Libary)

Figura 23 - Transmissão da Mensagem de Navegação com espalhamento C/A na L1.

Figura 24 - Erro do GPS devido a Troposfera e lonosfera. 53

Figura 25 - Erro do GPS devido a múltiplos Caminhos.

Figura 26 - Boa distribuição espacial dos satélites. 56

Figura 27 - Má distribuição espacial dos satélites. 56

Figura 28 - Cadeia escondida de Markov que caracteriza a evolução do sistema.

Figura 29 - Densidade condicional da posição baseada no valor medido z1. (MAYBECK et. at., 1979)

Figura 30 - Densidade condicional da posição baseada no valor medido $z_{2}$. (MAYBECK et. at., 1979)

Figura 31 - Densidade condicional da posição baseada nos valores medidos $z_{2}$ e $z_{2}$. (MAYBECK et. at., 1979)

Figura 32 - Simulação do Filtro de Kalman com $\mathrm{W}=(0,01)^{2}$.

(WELCH e BISHOP et. al., 2006)

Figura 33 - Simulação do Filtro de Kalman com $\mathrm{W}=(0,1)^{2}$.

(WELCH e BISHOP et. al., 2006)

Figura 34 - Simulação do Filtro de Kalman com $W=(1)^{2}$.

(WELCH e BISHOP et. al., 2006)

Figura35 - Transformação não-linear de uma distribuição com

pequena variância. (THRUN; BURGARD; e FOX et. al., 2005)

Figura 36 - Transformação não-linear de uma distribuição com

grande variância. (THRUN; BURGARD; e FOX et. al., 2005)

Figura 37 - Transformação através de um ponto com pequena nãolinearidade local. (THRUN; BURGARD; e FOX et. al., 2005)

Figura 38 - Transformação através de um ponto com grande nãolinearidade local. (THRUN; BURGARD; e FOX et. al., 2005)

Figura 39 - Aproximações à superfície da Terra.

Figura 40 - Sistema de Coordenadas ECEF. (Department

of Defense World Geodetic System 1984 et. al., 2000)

Figura 41 - Representação do Ponto em Latitude, Longitude e 
Altura.

Figura 42 - Sistema de Coordenadas ECI 79

Figura 43 - Sistema de Coordenadas ENU 79

Figura 44 - Wander Azimuth 80

Figura 45 - Coordenadas do Corpo 81

Figura 46 - Coordenadas da Medida 81

Figura 47 - Diagrama de Blocos da Navegação Inercial 83

Figura 48 - Rotação do sistema (BOSE. et. al., 1982) 91

Figura 49 - Velocidade angular em torno do eixo E e N. 91

Figura 50 - Velocidade Angular no sistema NAV 92

Figura 51 - Velocidade Angular total. (BOSE et. al., 1982) 93

Figura 52 - Ângulos de Euler da Matriz de Rotação $C_{e}^{n}$.

(BOSE et. al., 1982) 95

Figura 53 - Erro na Posição Angular. (BOSE et. al., 1982) 102

Figura 54 - Erro na Posição Angular Zoom. (BOSE et. al., 1982) 102

Figura 55 - Diagrama de Transformação do Erro na

Posição Angular.

103

Figura 56 - Diagrama de Blocos da Propagação do Erro

na Navegação Inercial 106

Figura 57 - Diagrama de Blocos da Fusão GPS/INS 115

Figura 58 - Diagrama de Blocos da Simulação 126

Figura 59 - Modelo Simplificado do Robô Ambiental Híbrido 127

Figura 60 - Diagrama de Blocos da Fusão GPS/INS 133

Figura 61 - Mapa Geo-referenciado do Percurso Medido

pelo GPS na Simulação. $\quad 137$

Figura 62 - Erro do Percurso Medido pelo GPS na Simulação. 137

Figura 63 - Mapa Geo-referenciado do Percurso Calculado pela

Navegação Inercial Clássica e com Orientação. 138

Figura 64 - Erro do Percurso Calculado pelo INS na Simulação. 138

Figura 65 - Mapa Geo-referenciado do Percurso Calculado

pela Fusão GPS/INS Clássica e com Orientação.

Figura 66 - Erro do Percurso Calculado pelo GPS/INS

na Simulação. 
Figura 67 - Gráfico do Erro da Velocidade Linear Total do

INS/GPS e do GPS. 141

Figura 68 - Gráfico do Erro da Velocidade Linear Total INS. 142

Figura 69 - Erro de Roll do INS Clássico na Simulação. 143

Figura 70 - Erro de Roll do INS com Orientação e do

GPS/INS na Simulação. 143

Figura 71 - Erro de Pitch do INS Clássico na Simulação. 144

Figura 72 - Erro de Pitch do INS com Orientação e do

GPS/INS na Simulação. 144

Figura 73 - Erro de Yaw do INS Clássico na Simulação. 145

Figura 74 - Erro de Yaw do INS com Orientação e do

GPS/INS na Simulação. 145

Figura 75 - Erro Total da Aceleração Linear na Simulação. 146

Figura 76 - Erro da Velocidade Angular na Simulação. 147

Figura 77 - Erro do Percurso na Simulação. 148

Figura 78 - Erro da Velocidade Linear Total na Simulação. 149

Figura 79 - Raiz do Erro Quadrático da Orientação na Simulação. 149

Figura 80 - Raiz do Erro Quadrático da Velocidade Angular na Simulação.

Figura 81 - Raiz do Erro Quadrático da Aceleração Linear na Simulação. 150

Figura 82 - Robô Ambiental Híbrido 152

Figura 83 - Sensor Inercial (Xsens) 152

Figura 84 - Sensor GPS (Garmin) 152

Figura 85 - Painel de Controle 1

Figura 86 - Painel de Controle 2

Figura 87 - Área de Testes na Floresta Amazônica 154

Figura 88 - Diagrama de Blocos do Experimento 155

Figura 89 - Mapa Geo-Referenciado das posições do Filtro

de Kalman, do INS e do GPS nos testes na Amazônia. 157

Figura 90 - Zoom do Mapa Geo-Referenciado das posições do

Filtro de Kalman, do INS e do GPS nos testes na Amazônia

Figura 91 - Corte 1 da Ampliação do Mapa Geo-Referenciado das 
posições do Filtro de Kalman, do INS e do GPS nos testes na Amazônia

Figura 92 - Corte 2 da Ampliação do Mapa Geo-Referenciado das posições do Filtro de Kalman, do INS e do GPS nos testes na Amazônia 158

Figura 93 - Erro de Altitude do INS nos testes da Amazônia 159

Figura 94 - Erro de Altitude do Filtro de Kalman nos testes da Amazônia 160

Figura 95 - Navegação do GPS e INS/GPS em torno da lagoa. 161

Figura 96 - Navegação do INS em torno da lagoa.

Figura 97 - Navegação do INS, INS/GPS e Real em torno da Lagoa. 163

Figura 98 - INS/GPS Clássico em torno da lagoa. 164

Figura 99 - Mapa da Falha do Sinal do GPS no Experimento na Lagoa.

Figura 100 - Latitude no Experimento na Lagoa. 166

Figura 101 - Longitude no Experimento na Lagoa. 166

Figura 102 - Vx no Experimento na Lagoa. 167

Figura 103 - Vy no Experimento na Lagoa. 167

Figura 104 - Roll no Experimento na Lagoa. 168

Figura 105 - Pitch no Experimento na Lagoa. 168

Figura 106 - Yaw no Experimento na Lagoa. 169

Figura 107 - Resultado experimental de (WANG et. al., 2006) 170

Figura 108 - Resultado experimental de (UMAR; TASHFEEN;

AIME; e NOURELDIN et. al., 2009) 171

Figura 109 - "Datasheet" Xsnes 177

Figura 110 - "Datasheet" GPS Parte 1

Figura 111 - "Datasheet" GPS Parte 2 


\section{Lista de tabelas}

Tabela 1 - Sistemas de GNSS existentes

Tabela 2 - Desvios padrão dos erros na estimativa de posição utilizando o sinal de GPS. (SCHRIEVER)

Tabela 4 - Parâmetros do WGS-84 (Department of Defense

World Geodetic System 1984 et. al, 2000) 76

Tabela 3 - Parâmetros do modelo do Robô Ambiental Híbrido 128

Tabela 4 - Fontes de Erro do Sensor Inercial (Xsens) 128

Tabela 5 - Diagonal da Matriz de Ruído do Processo (V) 134

Tabela 6 - Diagonal da Matriz de Ruído do Medida Implementação Clássica (W)

Tabela 7 - Diagonal da Matriz de Ruído do Medida Implementação com Orientação (W) 134

Tabela 8 - RMS da Simulação

Tabela 9 - Diagonal da Matriz de Ruído do Processo

Experimental (V)

Tabela 11 - Diagonal da Matriz de Ruído da Medida

Experimental (W) 